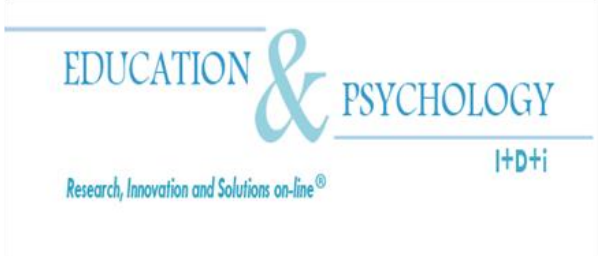

\title{
Aspectos motivacionales, volitivos y metacognitivos del aprendizaje autorregulado
}

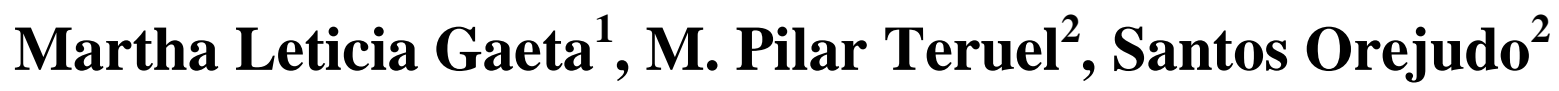

${ }^{1}$ Doctorado en Pedagogía, Universidad Popular Autónoma del Estado de Puebla, Puebla

${ }^{2}$ Facultad de Educación, Universidad de Zaragoza, Zaragoza

\section{México, España}

Correspondencia: Martha Leticia Gaeta. 17 Sur 901 Colonia Santiago, 72410 Puebla, México. E-mail: marthaleticia.gaeta@upaep.mx

C) Education \& Psychology I+D+i and Editorial EOS (Spain) 


\section{Resumen}

Introducción. Hoy en día uno de los principales objetivos de la educación es ayudar a los estudiantes a ser autónomos y eficaces. Esto sólo es posible en la medida en que los alumnos autorregulen su propio aprendizaje. Por lo tanto, el objetivo de este estudio fue estudiar algunos de los factores contextuales y personales que facilitan este proceso.

Método. Los participantes fueron 604 estudiantes de Educación Secundaria en una ciudad al noroeste de España. Se utilizó el Modelado de Ecuaciones Estructurales (SEM) para determinar qué tan bien el modelo teórico propuesto se ajustaba a los datos de la investigación.

Resultados. El análisis de las relaciones entre las variables de modelo revelan los siguientes resultados: la percepción de los estudiantes de la estructura del aula es una condición importante para el desarrollo de su orientación personal a metas. La orientación a meta parece conducir a los alumnos a asumir la responsabilidad (o no) con la persistencia y perseverancia necesarias para lograr los objetivos definidos por su orientación motivacional, a través del control de la motivación y la emoción. Este esfuerzo y perseverancia para el logro de la meta a su vez tiene un efecto positivo sobre el uso de estrategias para controlar y dirigir sus procesos mentales para la autorregulación del aprendizaje.

Discusión y conclusiones. Este estudio mostró la importancia de la percepción que los estudiantes tienen de la estructura de metas de aula, a fin de involucrarse en su propio aprendizaje y la posibilidad de aumentar el uso de estrategias metacognitivas, para la regulación automática de aprendizaje, mediante el uso de estrategias volitivas.

Palabras clave: motivación, voluntad, metacognición, aprendizaje autorregulado 


\title{
Motivational, volitional and metacognitive aspects of self regulated learning
}

\begin{abstract}
Introduction. Today one of the main objectives of education is to help students to become autonomous and efficient learners. This is only possible to the extent that students selfregulate their own learning. Hence, the purpose of this study was to study some contextual and personal factors that facilitate this process.
\end{abstract}

Method. Participants were 604 high-school students from a northwestern city in Spain. Structural Equation Modeling (SEM) was utilized to determine how well the proposed theoretical model fit the research data.

Results. Analyses of the relationships between the model variables reveal the following results: students' perception of the classroom structure is an important condition for the development of his or her personal goal orientation. Goal orientation appears to lead students to take responsibility (or not) with the persistence and perseverance required to achieve the goals defined by their motivational orientation, by controlling motivation and emotion. This effort and persistence for goal achievement has in turn a positive effect on the use of strategies to control and direct his or her mental processes for the self-regulation of learning.

Discussion and conclusions. This study showed the importance of students' perception of the classroom goal structure to get involved in their own learning, and the possibility to increase the use of metacognitive strategies, for the self- regulation of learning, through the use of volitional strategies.

Key words: Motivation, volition, metacognition, self-regulated learning 


\section{Introducción}

El estudio de los factores que influyen en el aprendizaje y logro de los estudiantes es uno de los temas a los que la investigación educativa ha dedicado su atención durante las últimas décadas. El énfasis actual está en la participación activa de los estudiantes en su propio aprendizaje a fin de que sean independientes, autónomos y auto-motivados. Cobra así, en este contexto, especial relevancia la autorregulación del aprendizaje, la cual centra su atención en cómo los estudiantes personalmente modulan su pensamiento, afecto y comportamiento, a través del uso de mecanismos específicos y meta-habilidades de apoyo hacia el logro de sus objetivos (Zimmerman, 1989).

En general, para que el aprendizaje significativo tenga lugar, los estudiantes deben estar motivados para aprender y poseer y movilizar las estrategias necesarias para regular su cognición (estrategias metacognitivas). También necesitan estrategias que les ayuden a persistir y mantener su esfuerzo hacia el logro de metas que mejoren su aprendizaje (estrategias volitivas). Además, los factores contextuales tienen un papel importante en facilitar o restringir la autorregulación.

En este contexto, la Teoría de Orientación a Metas constituye una línea de investigación actual en el estudio de la motivación de los alumnos hacia el aprendizaje. Las orientaciones a meta se refieren a los propósitos que guían a los individuos para iniciar y desarrollar acciones en situaciones de logro (Pintrich y Schunk, 2006). Además de reflejar los criterios mediante los cuales las personas juzgan su ejecución en la tarea y su éxito o fracaso respecto a la consecución de la meta (Urdan, 2004). Tradicionalmente, en la investigación de la orientación a metas, se diferencian dos tipos principales de metas que los estudiantes pueden adoptar en el contexto académico, de acuerdo a cómo perciben su nivel de competencia: metas de aprendizaje y metas de rendimiento (Kaplan y Midgley, 1999).

Algunos investigadores (Midgley et al., 2000) han considerado a ambas orientaciones como más complejas, tanto desde una perspectiva teórica como a partir los resultados empíricos. Proponen que la orientación al aprendizaje se contrapone con la orientación al rendimiento (ambas de aproximación positiva), e incluyen además una nueva meta de rendimiento, denominada de evitación a la tarea, que incluye el componente de evitación. La meta de rendi- 
miento de aproximación se centra en querer demostrar una mayor competencia. Mientras que la meta de rendimiento de evitación se enfoca en buscar evitar parecer incompetente.

Varios estudios han encontrado, desde esta perspectiva, que el aprendizaje autorregulado puede ser adquirido a través de metas de aprendizaje (Pintrich, 1999). Sin embargo, existen diferentes resultados en cuanto a la adopción de metas para demostrar la habilidad (Muis, Winne y Edwards, 2009) y resultados negativos cuando se adoptan metas para evitar demostrar falta de habilidad (Urdan, 2004). Por lo que, de acuerdo a la meta que el estudiante adopte, él o ella evalúa las situaciones de aprendizaje y las tareas de manera diferente (Tapola y Niemivirta, 2008), se involucra en las tareas con diferentes expectativas, y explica los resultados obtenidos de forma diferente (Alonso Tapia, 1992).

De acuerdo con la Teoría de Orientación a Metas, las diferentes representaciones mentales que los estudiantes generan sobre los tipos de metas que pretenden alcanzar dentro del contexto educativo (orientación personal a metas) se efectúan bajo la influencia de factores externos tales como la planificación del profesor, el nivel de demanda y la organización de la clase (Pintrich y Schunk, 2006). Dos tipos generales de ambientes tienen lugar en el aula: aprendizaje y rendimiento. Una estructura de aprendizaje se ha definido como el ambiente educativo que enfatiza el aprendizaje y el esfuerzo. En un ambiente de aprendizaje prevalecen los auto-estándares de desempeño y la idea de que las nuevas habilidades y el conocimiento se adquieren mediante el involucramiento en situaciones de aprendizaje que implican reto. En contraste, en una estructura de rendimiento existe un fuerte énfasis en las recompensas externas; se enfatiza la habilidad del estudiante relativa al desempeño de otros (Ames, 1992).

Desde esta perspectiva, varios estudios han encontrado que las percepciones de los estudiantes de la estructura de metas del aula están relacionadas positivamente con sus metas de logro personales hacia estas clases (Ames, 1992; Gaeta, 2006) y las orientaciones personales a meta a su vez predicen significativamente su nivel de persistencia e involucramiento en la tarea (Radosevich, Vaidyanathan, Yeo y Radosevich, 2004). Específicamente, las percepciones de una estructura de aprendizaje están relacionadas positivamente a patrones más adaptativos de aprendizaje tales como el uso efectivo de estrategias de aprendizaje, así como a un involucramiento en la clase, motivación, esfuerzo, estados afectivos y eventualmente al logro académico (Sideridis, 2005). En contraste, una estructura de metas de rendimiento se ha asociado con patrones de aprendizaje negativos (Ryan, Gheen y Midgley, 1998). 
Dependiendo de que la meta que se persiga sea mejorar la habilidad (orientación aprendizaje), demostrar la habilidad (orientación al rendimiento) o evitar parecer incompetente (orientación al rendimiento-evitación), se dan diferencias en la forma de pensar y actuar en la realización de una tarea (Midgley et al., 2000). Consecuentemente, existirán variaciones respecto al procesamiento cognitivo y a los procesos de regulación del aprendizaje.

La autorregulación a nivel cognitivo implica, por un lado, conocer y manejar un número de estrategias cognitivas y metacognitivas para llevar a cabo las tareas de estudio y, por otro lado, conocerse a uno mismo como procesador de información. La autorregulación implica además conocer los requerimientos de cada tarea para poder planificar, establecer metas, organizarse, auto-controlarse y evaluarse durante el aprendizaje (Roces y González, 1998).

Conceptualmente, de acuerdo con la literatura de la psicología contemporánea, la metacognición consiste en el conocimiento y la regulación de los propios procesos cognitivos. El conocimiento metacognitivo se refiere principalmente a la información que una persona posee sobre sus procesos cognitivos. En contraste, la regulación de la cognición incluye: los procesos de establecimiento de metas, la planificación de actividades, la supervisión durante el aprendizaje y la revisión y evaluación de los resultados (Brown, 1987). Forman parte de lo que se ha denominado "estrategias metacognitivas" (Lompscher, 1994; en Efklides, 2006).

Como hemos visto, la motivación es un factor esencial para el logro y aprendizaje de los estudiantes (Pintrich y Schunk, 2006). Sin embargo, las metas académicas a menudo requieren tiempo para lograrse, pudiéndose presentar varias situaciones que dificultan la acción requerida para lograr los objetivos deseados, así como fluctuaciones en la motivación debidas a actitudes y estados emocionales (Husman, McCann y Crowson, 2000). Por ello, ante la disminución en la motivación, la habilidad de los estudiantes para utilizar estrategias que les ayuden a dirigir su motivación hacia la acción, en la dirección de la meta propuesta, es un aspecto central del aprendizaje autorregulado (Wolters y Rosenthal, 2000).

A fin de que la motivación y el interés de los estudiantes en el aprendizaje se mantengan, necesitan ser capaces de autorregular su actividad durante el proceso de aprendizaje (Corno y Rohrkemper, 1985, en Alonso Tapia, 1992). En este sentido, la volición, que tiene la intención de regular el esfuerzo, es fundamental para el desempaño de los estudiantes, especialmente cuando el logro de los objetivos exige de concentración y esfuerzo durante largos 
periodos de tiempo (Heckhausen y Kuhl, 1985). Desde este enfoque se enfatiza el papel de la voluntad como fuerza impulsora que mueve a la persona y le permite ejecutar sus decisiones y proteger sus estados psicológicos ante las otras alternativas, pensamientos y emociones no deseadas. Los modelos actuales de autorregulación del aprendizaje proponen que las estrategias volitivas para mantener la motivación y el esfuerzo hacia el logro de los objetivos, así como para controlar las emociones negativas, están interrelacionadas y participar conjuntamente en la autorregulación de aprendizaje (Pintrich, 2000a).

Esta variable mediadora (la volición) ha sido incluida también en otros modelos cognitivo motivacionales. En este contexto, varios estudios han encontrado una relación entre la orientación a metas de aprendizaje y el uso de estrategias para aumentar la motivación y el esfuerzo (Bartels, Ma-Gun-Jackson y Kemp, 2009; Radosevich et al., 2004). Diversos estudios han demostrado también el papel mediador de las estrategias volitivas en el proceso de aprendizaje, encontrando que la volición y el uso de estrategias metacognitivas influyen sobre un mejor rendimiento académico (Wolters, 1998; 2000).

\section{El presente estudio}

Las características únicas de cada ambiente académico, combinadas con las de los propios estudiantes, hacen del aprendizaje un gran reto. Específicamente, en la enseñanza secundaria, los estudiantes no sólo tienen que afrontar sus cambios emocionales y biológicos, sino que también deben ser capaces de manejar diferentes tareas de varios profesores, en un programa de estudio más amplio donde se espera, además, que se involucren en un mayor tiempo de estudio independiente. Para poder cumplir con estas expectativas, los estudiantes necesitan tener un repertorio de estrategias de autorregulación de las cuales poder disponer y usar (Suárez y Fernández, 2011). De ahí la necesidad de capacitar a los estudiantes hacia un aprendizaje autónomo, mediante el uso de diferentes estrategias que controlan muchos aspectos de su cognición, motivación y afecto a fin de cumplir con sus metas académicas.

En este estudio partimos de la premisa básica que la metacognición es un constructo central en los procesos de autorregulación del aprendizaje (Pintrich, Smith, García y McKeachie, 1991). Los alumnos autorregulados utilizan sus conocimientos metacognitivos para regular su aprendizaje eficazmente y, a su vez, la regulación de su propio aprendizaje puede llevarlos a adquirir nuevos conocimientos relacionados con la tarea, las estrategias que deben manejar y sus propios recursos de aprendizaje (Pozo et al., 2006). Desde este marco, las estra- 
tegias metacognitivas, que incluyen estrategias de planificación, supervisión y regulación, ayudar a los estudiantes en el control y regulación de su cognición y, por tanto, les ayudan a promover un aprendizaje independiente (Pintrich et al., 1991).

Además, las diferencias del propio desarrollo, individuales y contextuales pueden interferir o apoyar los esfuerzos hacia la autorregulación. Aunque los estudiantes de diferentes edades pueden tener experiencias metacognitivas, las cuales mejoran con la edad, es la capacidad para tomar ventaja de ellas lo que ayuda a los alumnos en la adquisición de conocimientos y habilidades. De ahí la importancia de que los adolescentes conozcan y controlen sus procesos cognitivos a fin de involucrarse cognitivamente. Asimismo, en la escuela los estudiantes persiguen múltiples metas, por lo que las metas de aprendizaje no siempre son adoptadas por los alumnos y a veces les resulta difícil mantener sus intenciones hacia el logro de sus metas de aprendizaje, incluso cuando han sido adoptadas (Boekaerts y Corno, 2005). Por lo que, el ser capaces de utilizar estrategias que les ayuden a mantener su motivación por aprender y controlar sus emociones en situaciones difíciles o ante metas alternas (control de voluntad) es de gran relevancia (McCann y Turner, 2004).

\section{Objetivos e hipótesis}

Con base en lo anterior, en este estudio proponemos que el uso de estrategias metacognitivas estará relacionado con diferentes variables motivacionales y volitivas. Las variables motivacionales incluyen las percepciones de la estructura del aula y las orientaciones personales a meta, y las variables volitivas comprenden las estrategias de control motivacional y emocional. Proponemos un conjunto de relaciones estructurales entre estos constructos; las percepciones de los estudiantes de la estructura de metas de aula tienen una influencia directa sobre su orientación a metas, y las estrategias volitivas - de control de motivacional y emocional - tienen un papel mediador entre las orientaciones a meta de los estudiantes y el uso de estrategias metacognitivas.

Los supuestos básicos del modelo y las relaciones entre las variables correspondientes se muestran en la Figura 1. Desde una perspectiva general, en el modelo de investigación se propone que: 
1. La estructura de metas de rendimiento y la estructura de metas de evitación incidirán positivamente sobre la orientación a metas de rendimiento.

2. La orientación a metas de rendimiento tendrá una asociación positiva con las estrategias metacognitivas.

3. La estructura de metas de aprendizaje tendrá una relación positiva con la orientación a metas de aprendizaje.

4. La orientación a metas de aprendizaje incidirá positivamente en las estrategias metacognitivas.

5. Las estrategias volitivas mediarán la relación entre las orientaciones a metas de aprendizaje y de rendimiento y las estrategias metacognitivas.

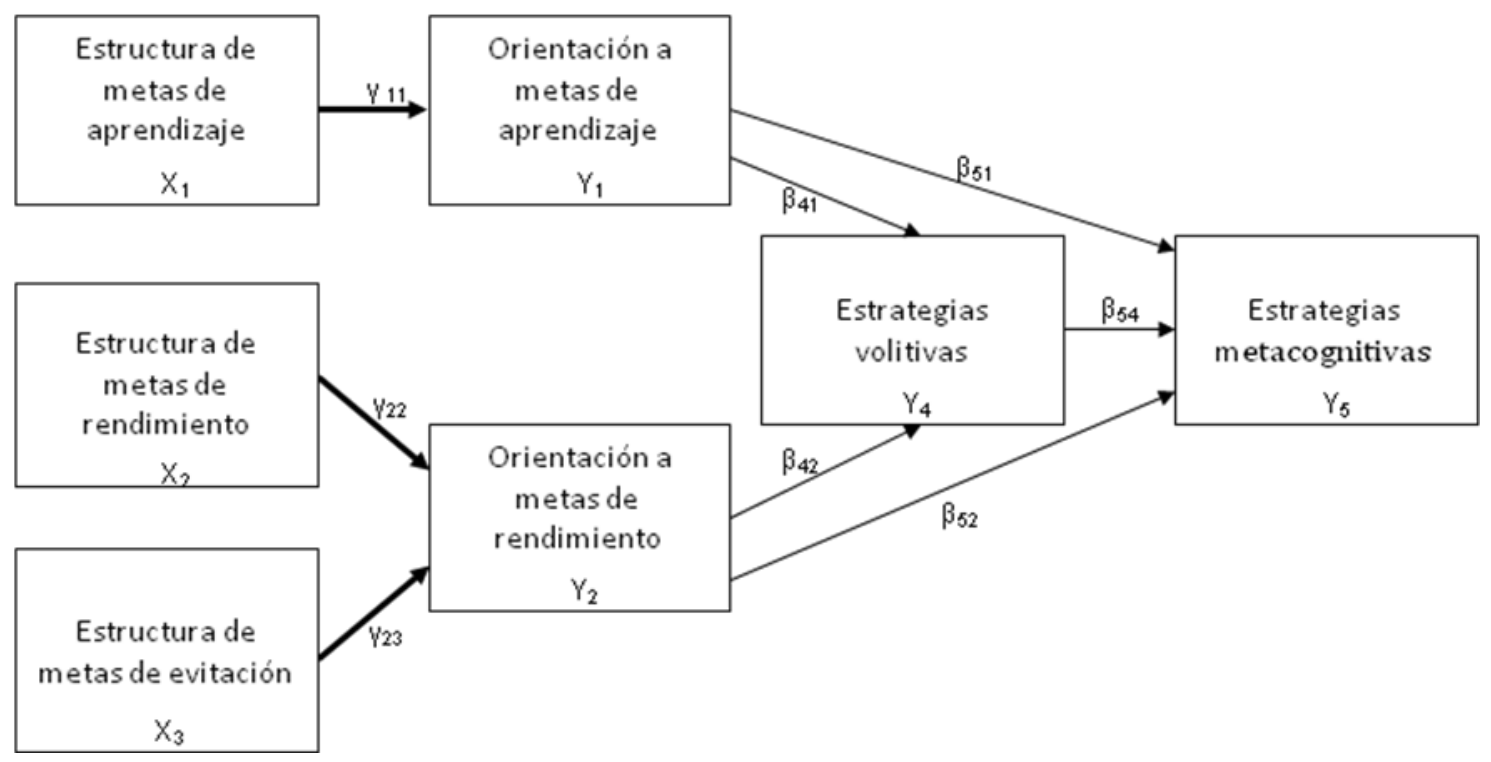

Nota: Las flechas gruesas indican los efectos $(\gamma)$ de las variables exógenas $(\mathrm{X})$ sobre las variables endógenas $(\mathrm{Y})$. Las flechas finas indican los efectos principales $(\beta)$ entre las variables $\mathrm{Y}$.

Figura 1. Representación gráfica del modelo propuesto.

Respecto a las estrategias volitivas, utilizamos una sola variable latente "volición", que integraba las estrategias de "fortalecimiento de la auto-eficacia", "las acciones para reducir el estrés" y "los incentivos con base negativa". Se usaron, además, dos mitades aleatorias como indicadores para todas las variables latentes del modelo, a fin de reducir el número de parámetros (MacCallum y Austin, 2000). Se utilizó un total de 14 indicadores y 7 variables latentes para medir el modelo estructural teórico. 


\section{Método}

\section{Participantes}

Participaron en el estudio 604 estudiantes de secundaria, de séptimo $(n=336)$ y décimo $(n=268)$ grados, de tres centros públicos y tres concertados de una ciudad noroccidental en España. La muestra consistió de 303 chicas y 301 chicos, entre 12 y 17 años de edad. Se utilizó un muestreo aleatorio estratificado en el estudio (importancia poblacional de cada zona en la ciudad y número y tipo de centro educativo: público y concertado).

\section{Instrumentos}

Las percepciones sobre la estructura de metas del aula por parte los estudiantes se midieron utilizando la sección correspondiente de la Escala de Patrones de Aprendizaje Adaptativo (EPAA; Midgley et al., 2000). Contiene tres subescalas: La escala "estructura de metas de aprendizaje" mide las percepciones de los estudiantes de que el propósito para involucrarse académicamente es desarrollar las habilidades y competencias (p. ej., "en nuestra clase, esforzarse es muy importante"). La escala "estructura de metas de rendimiento " mide la percepciones de que el propósito es demostrar las habilidades y competencias (p. ej., "en nuestra clase, obtener buenas calificaciones es el principal objetivo"). La escala "estructura de metas de evitación" mide las percepciones de que el propósito es evitar demostrar incompetencia (p. ej., "en nuestra clase, el mostrar a otros que no eres malo en el trabajo de clase es realmente importante"). Midgley et al. (2000) obtuvieron los siguientes coeficientes de confiabilidad ( $\alpha$ de Cronbach) para cada una de las subescalas: estructura de metas de aprendizaje (.76), estructura de metas de rendimiento (.70) y estructura de la metas de evitación (.83). En nuestra muestra, obtuvimos coeficientes ( $\alpha$ de Cronbach) para cada escala de.75, .66 y.71, respectivamente, y la estructura factorial de la escala utilizando nuestros datos coincide completamente con la obtenida en el instrumento original.

Las orientaciones a meta de los alumnos fueron evaluadas por medio de la sección correspondiente de la Escala de Patrones de Aprendizaje Adaptativo (EPAA; Midgley et al., 2000). El cuestionario proporciona una evaluación de tres tipos generales de metas académicas: la escala "orientación a metas de aprendizaje" indica el involucramiento de los estudiantes en la tarea para desarrollar su competencia (p. ej., "hago mis trabajos escolares porque estoy interesado en ello"). La escala "orientación a metas de rendimiento " es indicativa de un estudiante que quiere demostrar su competencia (p. ej., "quiero hacerlo mejor que los otros 
estudiantes de esta clase"). La escala "orientación a metas de rendimiento-evitación" indica que el propósito de los estudiantes es evitar parecer incompetentes (p. ej., "la razón por la que hago mi trabajo es para que otros no piensan que soy tonto"). Midgley et al. (2000) obtuvieron los siguientes coeficientes de confiabilidad ( $\alpha$ de Cronbach) para cada una de las subescalas: orientación a metas de aprendizaje (.85), orientación a metas de rendimiento (.89) y orientación a metas de rendimiento-evitación (.74). En el presente estudio, tras el análisis factorial exploratorio, en lugar de los tres factores previstos, sólo surgieron dos factores: orientación a metas de aprendizaje ( $\alpha$ de Cronbach $=.78)$ y orientación a metas de rendimiento-evitación $(\alpha$ de Cronbach $=.83$ ); a este último hemos designado con el término "orientación a metas de rendimiento". Los coeficientes de confiabilidad obtenidos nos llevaron a considerar la escala un instrumento con índices bastante satisfactorios.

Para evaluar el uso de estrategias metacognitivas hemos utilizado la escala correspondiente del Cuestionario de Estrategias de Motivación para el Aprendizaje (CEMA II; Pintrich et al., 1991). La escala de estrategia metacognitivas evalúa las estrategias utilizadas por un estudiante para controlar y regular su propia cognición (p. ej., "cuando estoy leyendo para esta clase me detengo de vez en cuando y repaso lo que he leído"). En el presente estudio, la confiabilidad de esta escala fue bastante alta $(\alpha$ de Cronbach $=.79)$ en comparación con el coeficiente de confiabilidad obtenido en la escala original $(\alpha$ de Cronbach $=.78)$.

El uso de estrategias volitivas se evaluó mediante el Inventario de Estrategias Volitivas Académicas (IEVA; McCann y Turner, 2004). Este instrumento mide el grado en que los estudiantes se involucran en estrategias de regulación motivacional para controlar su motivación y estados emocionales cuando inician y buscan mantener la acción ante los requerimientos académicos. La escala de "fortalecimiento de la auto-eficacia" representa el control conductual en el que los estudiantes se alientan sobre su capacidad para realizar las tareas previstas (p. ej., "me digo a mí mismo que puedo hacerlo"). La escala de "acciones para reducir el estrés" representa las acciones que realizan los estudiantes para reducir el estrés producido por otras alternativas atractivas (p. ej., "suelo usar alguna técnica de relajación para concentrarme mejor en mis estudios"). La escala de "incentivos con base negativa" representa el intento de los alumnos por recordarse a sí mismos sus metas e incrementar su motivación para cumplir con los compromisos académicos (p. ej., "pienso en el tipo de trabajo/carrera en el que puedo acabar si fracaso en la escuela). En nuestro estudio, los coeficientes de confiabilidad ( $\alpha$ de Cronbach) para cada una de las escalas son: fortalecimiento de la auto-eficacia (.75), acciones 
para reducir el estrés (.61) e incentivos con base negativa (.58). La confiabilidad para el cuestionario total es bastante satisfactoria $(\alpha$ de Cronbach $=.81$ ) comparada con la del instrumento original ( $\alpha$ de Cronbach $=.87$ ).

\section{Procedimiento}

Todos los instrumentos fueron traducidos y adaptados al contexto español, contando con la revisión por expertos. El MSLQ había sido traducido y adaptado para muestras de estudiantes universitarios españoles (Roces, Tourón y González, 1995), por lo que sólo se realizó una revisión semántica para su aplicación en muestras a nivel secundaria. Los instrumentos de evaluación adaptados fueron administrados a los estudiantes en sus aulas, en una sola sesión durante el horario académico. Se aseguró a todos los estudiantes que sus respuestas serían confidenciales y se les animó a preguntar en caso de que hubiese preguntas poco claras.

\section{Análisis de los datos}

A fin de validar la estructura factorial de los instrumentos usados para el presente estudio, se realizó un Análisis Factorial Confirmatorio (AFC), mediante el programa LISREL 8.8 (Jöreskog y Sörbom, 2006). Para la estimación de los parámetros se utilizó el método de máxima verosimilitud; todos los análisis se llevaron a cabo con matrices de covarianza.

\section{Resultados}

\section{Análisis preliminares}

Se llevó a cabo un Análisis Factorial Confirmatorio (AFC) a fin de replicar la estructura factorial de los instrumentos utilizados en el estudio. Los índices de ajuste mostrados en la Tabla 1 indican un buen ajuste del modelo para las estructuras factoriales; se recomienda que los valores RMSEA estén por debajo de 0.10 para un ajuste moderado, por debajo de 0.05 para un buen ajuste, y por debajo de 0.01 para un excelente ajuste de los datos (Hair, Anderson, Tathaw y Black, 2000), se recomiendan valores superiores a 0.90 para un buen ajuste de GFI, AGFI, NFI, TLI y CFI (Jaccard y Wan, 1996) y la razón x2/g.l. por debajo de 5 se considera aceptable y por debajo de 3 indica un buen ajuste de los datos (Bollen1989). 
Tabla 1. Análisis Factorial Confirmatorio (AFC) de los instrumentos usados en el estudio (n=604).

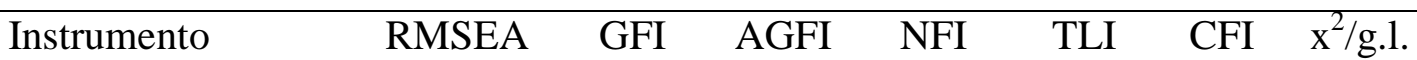

\begin{tabular}{lccccccc}
\hline $\begin{array}{l}\text { Percepciones sobre la Es- } \\
\text { tructura de Metas del Aula }\end{array}$ & .055 & .967 & .946 & .923 & .931 & .949 & 2.83 \\
$\begin{array}{l}\text { Orientaciones Personales a } \\
\text { Metas de Logro }\end{array}$ & .075 & .963 & .935 & .925 & .918 & .941 & 4.39 \\
$\begin{array}{l}\text { Estrategias de Motivación } \\
\text { para el Aprendizaje }\end{array}$ & .040 & .979 & .965 & .937 & .957 & .968 & 1.98 \\
Estrategias Volitivas & .052 & .952 & .934 & .876 & .893 & .912 & 2.61 \\
Académicas & & & & & & & \\
\hline
\end{tabular}

En general, los índices de ajuste, así como la significacancia de los parámetros y los valores medios de los errores estandarizados, indican que la estructura propuesta para todos los instrumentos no pueden ser rechazada (Hu y Bentler, 1995). Por lo tanto, todos los cuestionarios presentan una estructura adecuada para análisis posteriores.

Un análisis de las correlaciones de orden cero, mostradas en la Tabla 2, proporciona evidencia de validez de nuestras medidas. En general, la mayoría de variables estuvieron correlacionadas positivamente con el uso de estrategias metacognitivas. Las dos excepciones a este patrón fueron la estructura de metas de rendimiento y la estructura de evitación. Asimismo, esta última fue la única variable que no se correlacionó significativamente con la orientación al aprendizaje y el uso de estrategias volitivas. La orientación a metas de aprendizaje y las estrategias volitivas tuvieron la correlación más fuerte respecto al uso de estrategias metacognitivas. 
Tabla 2. Correlaciones entre las percepciones de la estructura del aula, la orientación a metas, $y$ el uso de estrategias volitivas y metacognitivas $(n=604)$.

\begin{tabular}{|c|c|c|c|c|c|c|c|}
\hline & 1 & 2 & 3 & 4 & 5 & 6 & 7 \\
\hline $\begin{array}{l}\text { 1. Estructura de me- } \\
\text { tas de aprendizaje } \\
\text { 2. Estructura de me- } \\
\text { tas de rendimiento } \\
\text { 3. Estructura de me- } \\
\text { tas de evitación }\end{array}$ & $\begin{array}{l}, 15^{* *} \\
-, 16^{* *} \\
\end{array}$ & , $26 * *$ & - & - & - & $\begin{array}{l}- \\
- \\
\end{array}$ & $\begin{array}{l}- \\
- \\
-\end{array}$ \\
\hline $\begin{array}{l}\text { 4. Orientación al } \\
\text { aprendizaje } \\
\text { 5. Orientación al } \\
\text { rendimiento }\end{array}$ & $\begin{array}{l}, 56 * * \\
, 02\end{array}$ & $\begin{array}{l}, 13 * * \\
, 23 * *\end{array}$ & $\begin{array}{l}-, 04 \\
, 56 * *\end{array}$ &, $14 * *$ & - & - & - \\
\hline $\begin{array}{l}\text { 9. Estretegias voliti- } \\
\text { vas }\end{array}$ &, $30 * *$ &, $12 * *$ & ,04 &, $47 * *$ &, $26 * *$ & - & - \\
\hline $\begin{array}{l}\text { 12. Estrategias me- } \\
\text { tacognitivas }\end{array}$ &, $30 * *$ & ,06 &,- 04 &, $56 * *$ & $13 * *$ &, $57 * *$ & - \\
\hline
\end{tabular}

Bondad de Ajuste del Modelo

Con base en los índices de ajuste, el modelo hipotético se ajusta muy bien a los datos. El RMSEA $=.061_{(.052 .071)}$ muestra un valor adecuado, el cual es corroborado por su intervalo de confianza. Los datos proporcionados por otros índices también ofrecen apoyo para aceptar el modelo propuesto en nuestro estudio $(\mathrm{NNFI}=.95$; $\mathrm{CFI}=.97$; GFI =. 95; $\mathrm{AGFI}=.92$; $\operatorname{SRMR}=.05 ; \mathrm{x} 2 / \mathrm{g} .1 .=3 \cdot 27)$.

A pesar del buen ajuste del modelo, los resultados mostraron que había un margen de mejora. Un examen detenido de la significación de los parámetros estimados y la hipotética relevancia de los no estimados (observada a través de los índices de modificación y los residuales estandarizados), nos llevó a modificar el modelo propuesto: se eliminó la ruta que muestra la relación hipotética entre la estructura de metas de rendimiento y la orientación a metas al rendimiento $(\gamma=.09 ; \mathrm{t}=1.62)$, así como la ruta que muestra la supuesta relación entre la orientación a metas de rendimiento y las estrategias volitivas $(\beta=.07 ; \mathrm{t}=1,58)$, ya que éstas no fueron significativas. Además, se incluyó para su estimación una ruta que muestra la relación entre las estrategias volitivas y la orientación al rendimiento $(\beta=.24 ; \mathrm{t}=4.07)$. El nuevo modelo contrastado mostró buenos resultados conceptualmente, respecto al ajuste del modelo $($ RMSEA =. 059(.050.068), NNFI =. 96; CFI =. 97; GFI =. 95; AGFI =. 92; SRMR =. 05; x2/d.f.=3.10) y a nivel de estimación de los parámetros específicos. 


\section{Evaluación de los parámetros individuales}

La Fig. 2 muestra los coeficientes de las relaciones propuestas entre las variables en el modelo. Sólo se incluyeron las relaciones significativas entre las variables, de acuerdo con el valor correspondiente de "t" ("t" es significativa por encima de 1.96, p =.05; Doménech, 1994). Los resultados obtenidos confirman total o parcialmente las hipótesis utilizadas para la construcción del modelo. En primer lugar, se confirma que la estructura de metas de evitación incide significativamente en la orientación a metas de rendimiento, sin embargo, este no es el caso para la estructura de rendimiento y la orientación al rendimiento. En segundo lugar, la orientación al rendimiento está asociada significativamente a las estrategias metacognitivas, pero no a las estrategias volitivas. En tercer lugar, la estructura de metas de aprendizaje está fuertemente relacionada a la orientación a metas de aprendizaje. En cuarto lugar, la orientación al aprendizaje incide significativamente en las estrategias metacognitivas. En quinto lugar, las estrategias volitivas tienen un efecto mediador significativo entre la orientación al aprendizaje y las estrategias metacognitivas. Además, las estrategias volitivas influyen en la orientación al rendimiento.

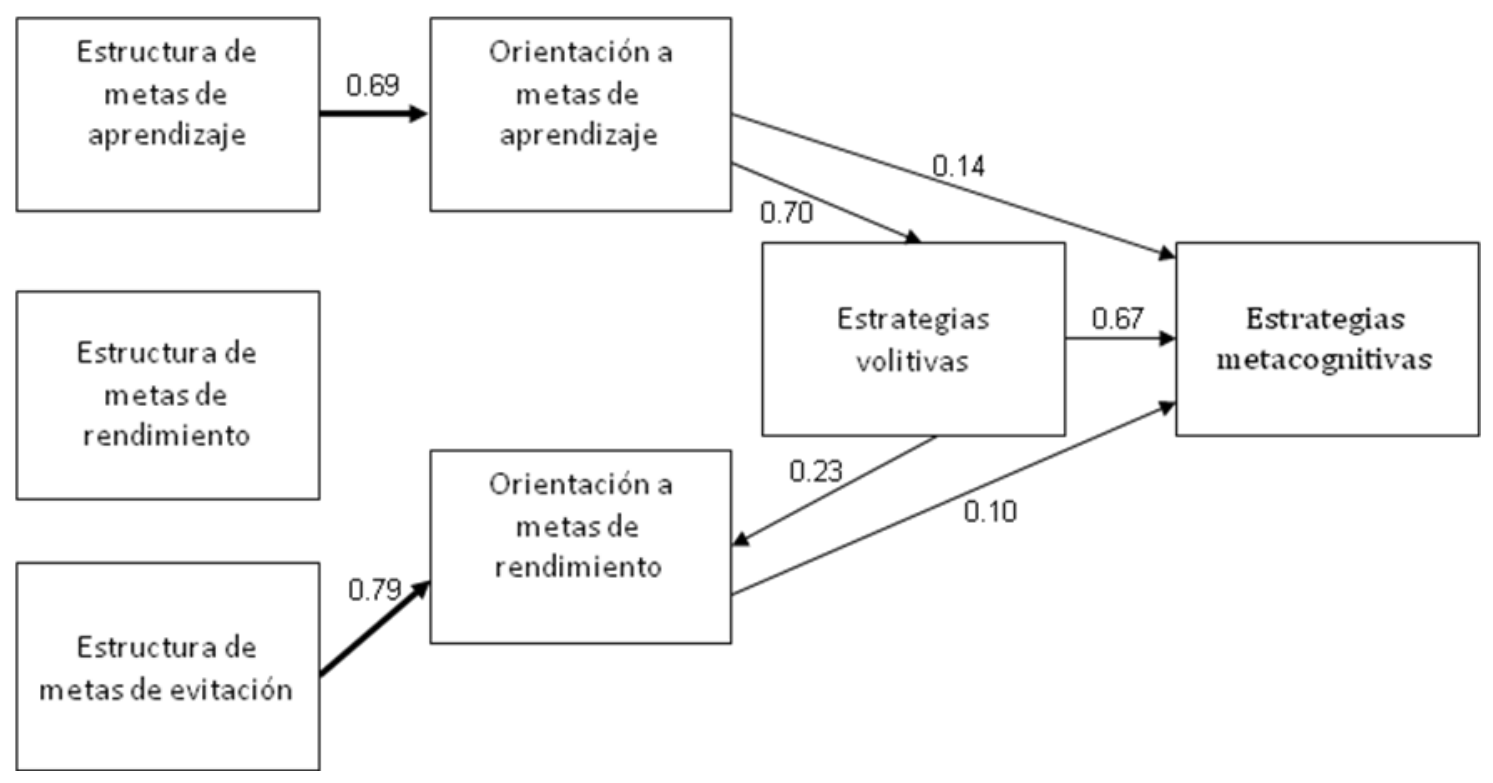

Nota: Todos los coeficientes estandarizados son estadísticamente significativos.

Figura 2. Coeficientes de las relaciones propuestas en el modelo (resultados estandarizados). 


\section{Discusión y Conclusión}

Una contribución del presente estudio es la evaluación empírica del modelo mostrado en la figura 1. De acuerdo con los criterios de evaluación del ajuste del modelo, los resultados obtenidos muestran un alto grado de congruencia entre el modelo teórico y los datos empíricos. Los análisis de las relaciones entre las variables del modelo revelan los siguientes resultados: la percepción de los estudiantes de la estructura del aula es una condición importante para el desarrollo de su propia orientación a metas (Ames, 1992). La orientación a metas parece llevar al estudiante a asumir (o no) la responsabilidad con la persistencia y la perseverancia necesarias para lograr los objetivos definidos por su orientación motivacional, controlando la motivación y la emoción (Bartels et al., 2009; Wolters y Rosenthal, 2000). Este esfuerzo y persistencia para el logro de sus metas tiene, a su vez, un efecto positivo sobre el uso de estrategias para controlar y dirigir sus procesos mentales para la autorregulación de su aprendizaje.

A partir del modelo mediacional se puede observar, en primer lugar, que la estructura de metas de evitación se relaciona significativamente con la orientación a metas de rendimiento. Sin embargo, contrario a nuestras expectativas, dicha orientación personal a metas no está relacionada con la estructura de metas de rendimiento. Esto indica que, en general, la mayoría de los estudiantes en este estudio percibe que el propósito para involucrarse en el trabajo académico no es tanto demostrar su competencia (p. ej., obtener buenas calificaciones), sino evitar demostrar su falta de competencia (p. ej., no ser el peor de la clase), lo que les lleva a compararse con los demás y evitar demostrar falta de habilidad (orientación al rendimiento).

La orientación al rendimiento se asocia, a su vez, con las estrategias metacognitivas, pero no con las estrategias volitivas, indicando que buscar ser mejor que otros o evitar parecer incompetente, no interfiere con el uso de estrategias metacognitivas y podría, incluso, promover su uso. Estos resultados coinciden con otros estudios en Educación Secundaria (Valle et al., 2006). Sin embargo, esta orientación a metas no parece promover el dedicar tiempo y esfuerzo en utilizar estrategias para perseverar en la tarea. Esto confirma la opinión de otros investigadores (Pintrich y Schunk, 2006), que afirman que los estudiantes orientados al rendimiento tienden a considerar al esfuerzo y a la habilidad como inversamente relacionados.

Otro importante hallazgo en este sentido es la influencia de las estrategias volitivas sobre la orientación a metas de rendimiento, lo que indica que el uso de estrategias volitivas - 
para fortalecer la auto-eficacia, reducir el estrés y establecer consecuencias por no actuar promueve, indirectamente, el involucramiento estratégico de los estudiantes, mediante la búsqueda de la valoración social positiva. Este resultado sugiere que aunque, en general, el comportamiento orientado al aprendizaje y el orientado al rendimiento pueden ser muy diferentes uno de otro, en situaciones donde los individuos están altamente seguros de sus habilidades, no habrá diferencias significativas entre las dos orientaciones (Millar, Behrens, Greene y Newman, 1993; en González, Valle, Nuñez y González-Pineda, 1996). Estos hallazgos nos conducen a una mayor investigación en este sentido, para una mejor comprensión de esta relación.

Encontramos, además, que la estructura de metas de aprendizaje está fuertemente relacionada con la orientación a metas de aprendizaje y esta última incide, a su vez, el uso de estrategias metacognitivas. Estos resultados coinciden con otras investigaciones (Ames y Archer, 1988), lo que indica que las percepciones de los alumnos de la estructura del aula, así como sus orientaciones personales a meta, son relevantes para su involucramiento cognitivo y su desempeño en el aula. Además, nuestros datos contribuyen a numerosos estudios (Urdan, 2004), mostrando que los estudiantes orientados al aprendizaje hacen un mayor uso de estrategias metacognitivas - actividades para planificar sus metas y auto-monitorear su comprensión del material - encontrando a la orientación al aprendizaje como más adaptativa y asociándola a una serie de mediadores de acción positiva.

Asimismo, de acuerdo con nuestras expectativas, encontramos que las estrategias volitivas tienen un papel mediador importante entre la orientación a metas de aprendizaje y las estrategias metacognitivas. Lo que indica que los estudiantes orientados al aprendizaje tienen más probabilidades de encontrar un vínculo fuerte entre su esfuerzo y sus resultados académicos, y a trabajar para reducir tanto las distracciones internas como externas (Pintrich y Schunk, 2006) mostrando mayores niveles de persistencia, en comparación con los estudiantes orientados al rendimiento. Estas estrategias de control motivacional y emocional producirán, como resultado, un mayor compromiso hacia el aprendizaje y al uso de estrategias de control cognitivo. Nuestra investigación sugiere además que cuando las tareas de aprendizaje no requieren un mayor uso de las estrategias volitivas, el esfuerzo y la persistencia son promovidos mediante la orientación a metas de aprendizaje. 
No obstante, existen algunas limitaciones en el presente trabajo que es necesario señalar. Aunque nuestros datos mostraron un ajuste adecuado al modelo propuesto, esto no significa que los datos no ajustarían con otra configuración de las variables. En segundo lugar, se necesitan más investigaciones mediante estudios longitudinales que permitan comprender los aspectos de causalidad. Además, hemos probado sólo un pequeño número de predictores teóricos de la autorregulación del aprendizaje y estos datos podrían apoyar también otros conjuntos de relaciones, tales como la influencia de las creencias de auto-eficacia, conocimientos previos, atribuciones cognitivas (véase Pintrich, 2000b) o expectativas (Suárez y Fernández, 2011).

En síntesis, a pesar de las limitaciones, este trabajo contribuye a una mejor comprensión de la importancia de la promoción de la autorregulación del aprendizaje de los estudiantes, a través de los entornos académicos de aprendizaje, la orientación a metas de aprendizaje y el uso de estrategias metacognitivas y volitivas. Este estudio también contribuye a un mejor entendimiento de cómo las estrategias que los estudiantes utilizan para mantener su esfuerzo en la tarea, y a regular sus emociones, favorece su involucramiento académico, al promover el uso de estrategias metacognitivas. 


\section{Referencias}

Alonso-Tapia, J. (1992). Motivar en la adolescencia: Teoría, evaluación e intervención. Madrid: Ediciones de la Universidad Autónoma de Madrid.

Ames, C. (1992). Classrooms: goals, structures, and student motivation. Journal of Educational Psychology, 84, 261-271.

Ames, \& Archer (1988). Achievement Goals in the Classroom: Students' Learning Strategies and Motivation Processes. Journal of Educational Psychology, 80(3), 260-267.

Bartels, J., Magun-Jackson, S., \& Kemp, A. (2009). Volitional Regulation and Self-Regulated Learning: An Examination of Individual Differences in Approach-Avoidance Achievement Motivation. Electronic Journal of Research in Educational Psychology, $7(2), 605-626$.

Boekaerts, M., \& Corno, L. (2005). Self-Regulation in the Classroom: A Perspective on Assessment and Intervention. Applied Psychology: An International Review, 54(2), 199231.

Bollen, K. A. (1989). Structural equations with latent variables. NY: John Wiley and Sons.

Brown, A. (1987). Metacognition, Executive Control, Self-Regulation and Other More Mysterious Mechanisms. En F. E. Weinert and R. H. Kluwe (Eds.), Metacognition, Motivation and Understanding, 65-116. Hillsdale, NJ: Erlbaum.

Corno, L. (1993). The best-laid plans: Modern conceptions of volition and educational research. Educational Researcher, 22(2), 14-22.

Doménech, J. M. (1994). Tablas de estadística (6ª edición). Barcelona: Herder.

Efklides, A. (2006). Metacognition and affect: What can metacognitive experiences tell us about the learning process? Educational Research Review, 1, 3-14.

Gaeta, M. L. (2006). Estrategias de autorregulación del aprendizaje: contribución de la orientación de meta y la estructura de metas del aula. Revista Electrónica Interuniversitaria de Formación del Profesorado, 9(1). Enlace web: http://www.aufop.com/aufop/home/ - Consultada el 30 de marzo, 2010.

González, R. C., Valle, A. A., Núñez, J. C., \& González-Pineda, J. A. (1996). Una aproximación teórica al concepto de metas académicas y su relación con la motivación escolar. Psicothema, 8(1), 45-61.

Hair, J., Anderson, R., Tatham, R., \& Black, W (2000). Análisis Multivariante (5 ed.). Madrid: Prentice Hall. 
Heckhausen, H., \& Kuhl, J. (1985). From wishes to action: the dead ends and short cuts on the long way to action. En M. Frese and J. Sabini (Eds.), Goal directed behavior: The concept of action in psychology (pp. 134-160). Hillsdale, NJ: Erlbaum.

Husman, J., McCann, E. J., \& Crowson, M. (2000). Volitional strategies and future time perspective: embracing the complexity of dynamic interactions. International Journal of Educational Research, 33, 777-799.

Jaccard, J. \& Wan, C. K. (1996). LISREL approaches to interaction effects in multiple regression (Quantitative Applications in the Social Sciences No. 114). Thousand Oaks, CA: Sage.

Jöreskog, K. G., \& Sörbom, D. (2006). LISREL 8,80. Chicago, IL: Scientific Software International Inc.

Kaplan, A., \& Midgley, C. (1999). The relationship between perceptions of the classroom goal structure and early adolescents' affect in school: the mediating role of coping strategies. Learning and Individual Differences, 11(2), 187-212.

MacCallum, R. C., \& Austin, J. T. (2000). Applications of structural equation modeling in psychological research. Annual Review of Psychology, 51, 201-226.

McCann, E. J., \& Turner, J. E. (2004). Increasing student learning through volitional control. Teachers College Record, 106(9), 1695-1714.

Midgley, C., Maehr, M. L., Hicks., L, Roeser, R., Urdan, T., Anderman, E. M., \& Kaplan, A. (2000). The Patterns of Adaptive Learning Survey (PALS). Ann Arbor: University of Michigan.

Muis, K. R., Winne, P. H., \& Edwards, O. V. (2009). Modern psychometrics for assessing achievement goal orientation: A Rash analysis. British Journal of Educational Psychology, 79, 547-576.

Pintrich, P. R. (1999). The role of motivation in promoting and sustaining self regulated learning. International Journal of Educational Research, 31, 459-470.

Pintrich, P. R. (2000a). Taking control of research on volitional control: challenges for future theory and research. Learning and Individual Differences, 11(3), 335-354.

Pintrich, P. R. (2000b). The role of goal orientation in self-regulated learning. En M. Boekaerts, P. R. Pintrich y M. Zeidner (Eds.), Handbook of Self-regulation (pp. 451502). San Diego, CA: Academic Press.

Pintrich, P. R., \& Schunk, D. H. (2006). Motivación en contextos educativos (M. Limón Trad.). Madrid: Prentice Hall. 
Pintrich, P. R., Smith, D., García, T., \& McKeachie, W. J. (1991). A manual for the use of the Motivated Strategies for Learning Questionnaire (MSLQ). Ann Arbor: University of Michigan.

Pozo, J. I., Scheuer, N., Pérez, M., Mateos, M., Martín, E., \& Cruz (2006). Nuevas formas de pensar la enseñanza y el aprendizaje. Las concepciones de profesores y alumnos. Barcelona: Grao.

Radosevich, D. J., Vaidyanathan, V. T., Yeo, S., \& Radosevich, D. M. (2004). Relating goal orientation to self regulatory processes: A longitudinal field test. Contemporary Educational Psychology, 29, 207-229.

Roces, C., Tourón, J. \& González, M. C. (1995). Motivación, estrategias de aprendizaje y rendimiento de los alumnos universitarios. Bordon, 47(1), 107-121.

Roces, C., \& González, M. C. (1998). Capacidad de autorregulación del proceso de aprendizaje. En J. A. González-Pineda and J. C. Núñez (Eds.), Dificultades del Aprendizaje Escolar (pp. 239-259). Madrid: Pirámide.

Ryan, A. M., Gheen, M. H., \& Midgley, C. (1998). Why Do Some Students Avoid Asking for Help? An Examination of the Interplay Among Students' Academic Efficacy, Teachers' Social-Emotional Role, and the Classroom Goal Structure. Journal of Educational Psychology, 90(3), 528-535.

Sideridis, G. D. (2005). Goal orientations, classroom goal structures, and regulation in students with and without learning disabilities: should we alter student's motivation, a classroom's goal structure, or both? En G. D. Sideridis and T. A. Citro (Eds.), Research to practice: Effective interventions in learning disabilities (pp. 193-219). Boston, MA: Learning Disabilities Worldwide.

Suárez, J., \& Fernández, A. (2011). A model of how motivational strategies related to the expectative component affect cognitive and metacognitive strategies. Electronic Journal of Research in Educational Psychology, 9(2), 641-658.

Tapola, A., \& Niemivirta, M. (2008). The role of achievement goal orientations in students' perceptions of and preferences for classroom environment.British Journal of Educational Psychology, 78, 291-312.

Urdan, T. (2004). Predictors of Academic Self-Handicapping and Achievement: Examining Achievement Goals, Classroom Goal Structures, and Culture. Journal of Educational Psychology, 96(2), 251-264. 
Valle, A., Cabanach, R. G., Rodríguez, S. M., Nuñez, J. C. y González-Pineda, J. A. (2006). Metas académicas, estrategias cognitivas y estrategias de autorregulación del estudio. Psicothema, 18(2), 165-170.

Wolters, C. A. (1998). Self-regulated learning and college students' regulation of motivation. Journal of Educational Psychology, 90(2), 224-235.

Wolters, C. A. (2000). The relation between high school students' motivational regulation and their use of learning strategies, effort, and classroom performance. Learning and Individual Differences, 3(3), 281-299.

Wolters, C. A., \& Rosenthal, H. (2000). The relation between students' motivational beliefs and their use of motivational regulation strategies. International Journal of Educational Research, 33, 801-820.

Zimmerman, B. J. (1989). A Social Cognitive View of Self-Regulated Academic Learning. Journal of Educational Psychology, 81(3), 329-339. 